doi: http://dx.doi.org/10.7124/bc.0009AE

\section{The role of the chromatin remodeling factor CHD1 in the global organization of Drosophila chromosomes}

\begin{abstract}
A.Tiutiunnik, I. Baranovskaya, Y. Kuchinskaya, Y. Gnennaya, A. Shalaev, A. Konev

NRC «Kurchatov Institute» - PNPI, Gatchina, Russia konev.alexander@gmail.com
\end{abstract}

Chromatin is organized into euchromatin and heterochromatin. Drosophila melanogaster also uses two systems of whole chromosome regulation: dosage compensation mediating the two fold up-regulation of male X-linked genes and the Painting of Fourth, regulating heterochromatic 4th chromosome. Both heterochromatin formation and dosage compensation are accompanied by global changes in chromatin structure. Evolutionary conserved cromatin assembly and remodeling factor CHD1 is an euchromatic protein which co-localizes in polytene chromosomes with the RNA polymerase II. The aim of this study was to analyze the role of Drosophila CHD1 in the control of global organization of polytene chromosomes. Methods: To study the action of CHD1 on global chromosome stucture, we examined the consequences of loss of CHD1 and of overexpression ATPase-dead or wild type CHD1 on polytene chromosome organisation in Drosophila larvae salivary glands. Results: In polytene chromosomes deprived of CHD1, we observed a specific bloating, blurring and shortening of the male $\mathrm{X}$ chromosome. Morphology of the female X chromosomes does not differ from autosomes and from the morphology of wild type chromosomes. In
Chd1 null mutant males, the maternally contributed CHD1 accumulates exclusively in $\mathrm{X}$-chromosome. In the wild type larvae we observed a specific enreachment of the CHD1 on the male X chromosome. Additional bands of CHD1 completely co-localize with the MSL proteins of the Dosage Compensation Complex (DCC), which associates specifically with the male $\mathrm{X}$ chromosome. The targeting of the CHD1 on the male $\mathrm{X}$ chromosome depends on the presence of a functional DCC. The loss of CHD1 results in preferential male lethality. Chd1 become essential in combination with deletion of one of two genes, encoding variant histone H3.3, His 3.3 B. An effect of Chd1 on male $\mathrm{X}$-chromosome morphology is increased by the His $3.3 \mathrm{~B}$ deficiency. GAL4-driven ectopic expression of wild type CHD1 or its ATPase-dead form leads to a strong decompactization of the specific regions of salivary gland polytene chromosomes in euchromatin. Immunostaining with antibodies to CHD1 and elongating RNA polymerase II reveals bright staining at all sites with an altered chromatin structure. Over-expression of wild type CHD1, but not of its ATPase-dead form also results in a strong decondensation of the chromocenter, where pericentric heterochromatin fuses, and of the 4th chromosome. Decondensed heterochromatin is brightly stained with antibodies to CHD1, where CHD1 does not co-localize with RNA Pol II and where heterochromatic protein HP1 and histone H1 are lost. Real time PCR analysis have revealed that the CHD1 over-expression increases the level of replication of X-chromosome heterochromatic repeats 1.688 satellite and Stellate, however does not affect the underreplication of the heterochromatic genes in autosomes. 
Conclusions: Here we show that the loss or over-expression of CHD1 severely and very specifically affect the global chromatin organization of Drosophila polytene chromosomes. Our finding suggests a new link between the organization of hyperactive chromatin of the male X - chromosome and of transcriptionally silent heterochromatin.

This work was partially supported by RFBR grant 15-0499583.

doi: http://dx.doi.org/10.7124/bc.0009AF

\section{High-resolution mapping of $\mathbf{A} / \mathbf{B}$ compartments and topologically associated domains on giant lampbrush chromosomes}

\section{Alla Krasikova, Anna Zlotina, Antonina Maslova, Polina Starshova, Tatiana Ku- likova}

Saint-Petersburg State University, Saint-Petersburg, Russia

alla.krasikova@gmail.com

Progress in studies aimed at "deciphering" the spatial architecture of the genome is determined by the development of several key technologies: the chromatin conformation capture, ultrahigh resolution optical microscopy and genomic locus imaging. At the same time, it remains unclear how the domains, determined by chromatin conformation capture technology, including topologically associated domains (TADs) and A/B compartments, are correlated with the chromatin domains detected at the cytological level. In the framework of this problem, a comprehensive study of the chro- matin domains of giant lampbrush chromosomes characteristic of the growing oocytes in birds, amphibians and reptiles, seems appropriate. Methods: Here we aimed at comparing the chromomeres - the main structural unit of lampbrush chromosome axes - and topologically associated domains and A/B compartments in domestic chicken (Gallus gallus domesticus), whose genome was the first among the deciphered avian genomes. In addition, earlier, using the full-genome Hi-C method a number of hierarchical structural domains, such as $\mathrm{A}$ and $\mathrm{B}$ compartments and TADs, were identified in chicken embryonic fibroblasts. Results: The results obtained allowed us to verify the hypothesis of the correspondence between the globular-loop chromatin domains of the interphase nucleus and the chromomereloop complexes of lampbrush chromosomes, as well as to shed light on the nature of the lampbrush chromosome chromomeres.

The research was supported by Russian Science Foundation (grant \# 19-74-20075). The work was partially performed using equipment of the Research Resource Center 'Molecular and cell technologies' of SPbU.

doi: http://dx.doi.org/10.7124/bc.0009B0

\section{Roles of actin family proteins in chromatin and nuclear functions}

Masahiko Harata

Laboratory of Molecular Biology, Graduate School of Agricultural Science, Tohoku University, Japan masahiko.harata.b6@tohoku.ac.jp

Genome functions are regulated by local chromatin structure and also by the association of individual genes with nuclear structures. As 